\title{
Impact of erosion on the nutrient and chemical profiles of agricultural soil in the Upper Rio Grande Valley, Jamaica
}

\author{
TIFFANY WALLACE ${ }^{1}$, ADRIAN SPENCE ${ }^{1 *}$, \\ DONOVAN CAMPBELL ${ }^{2}$, AND DAVID BARKER ${ }^{2}$ \\ ${ }^{1}$ International Centre for Environmental and Nuclear \\ Sciences, University of the West Indies, Kingston 7, \\ Jamaica (*adrian.spence02@uwimona.edu.jm) \\ ${ }^{2}$ Department of Geography and Geology, University of the \\ West Indies, Kingston 7, Jamaica
}

Land is a critical resource, providing food, feed, fiber, timber, energy, and many other ecosystem functions and services, including the regulation of climate. However, these critical functions and services have become increasing vulnerable to climate change and land degradation in the anthropocene. For instance, soil erosion and nutrient loss represent a formidable threath to food security and sustainable agriculture, especially in the global south.

Here we report on the use of gamma-ray emitting radionuclides $\left({ }^{7} \mathrm{Be},{ }^{137} \mathrm{Cs}\right.$ and $\left.{ }^{210} \mathrm{~Pb}\right)$ and a suite of macro and trace elements to determine sediment transport and the fate of soil nutrients in rural farming communities of the Upper Rio Grande Valley, Jamaica. Using the standardised PC-based conversion models, quantitative estimates of erosion and depositional rates are computed.

Preliminary results from gamma-ray emitting radionuclides indicate that erosion rates for managed land is $13.65 \mathrm{tha}^{-1} \mathrm{a}^{-1}$, while unmanaged land is $-0.48 \mathrm{t} \mathrm{ha}^{-1} \mathrm{a}^{-1}$. The relative uniformity of the radionuclide inventory of the managed land $\left(17.25 \pm 5.51 \mathrm{~Bq} \mathrm{~kg}^{-1}\right)$ confims medium to high tillage intensity. Whereas, an exponential decrease in the inventory of the unmanaged land confirms its protected status. Additionally, the concentrations of selected macro and trace elements vary widely for managed and unmanaged land. For example the concentrations of the macro element calcium range between $0.5 \% \pm 0.38$ and $0.24 \% \pm 0.18$ for managed and unmanaged land respectively. Similarly for trace elements, the concentrations vary widely as indicated by chromium $168.88 \mathrm{mg} / \mathrm{kg} \pm 30.44$ (managed land) and 349.39 $\mathrm{mg} / \mathrm{kg} \pm$ (unmanaged land). Trends for macro and trace elements for other selected elements are similar. Results also indicate a strong positive correlation between soil organic matter content and the radionuclide inventories. 